\title{
Mild Solutions of Neutral Semilinear Stochastic Functional Dynamic Systems with Local Non-Lipschitz Coefficients
}

\author{
Feng Jiang \\ School of Statistics and Mathematics, Zhongnan University of Economics and Law, Wuhan 430073, China \\ Correspondence should be addressed to Feng Jiang; fjiang78@gmail.com
}

Received 12 March 2013; Accepted 27 June 2013

Academic Editor: H. Srivastava

Copyright (C) 2013 Feng Jiang. This is an open access article distributed under the Creative Commons Attribution License, which permits unrestricted use, distribution, and reproduction in any medium, provided the original work is properly cited.

Semilinear stochastic dynamic systems in a separable Hilbert space often model some evolution phenomena arising in physics and engineering. In this paper, we study the existence and uniqueness of mild solutions to neutral semilinear stochastic functional dynamic systems under local non-Lipschitz conditions on the coefficients by means of the stopping time technique. We especially generalize and improve the results that appeared in Govinadan (2005), Bao and Hou (2010), and Jiang and Shen (2011).

\section{Introduction}

Semilinear stochastic dynamic systems in a separable Hilbert space often model some evolution phenomena arising in physics, biology engineering, and so forth [1]. Recently, for the case where the coefficients satisfy the global Lipschitz condition and the linear growth condition, many results are known [1-3]. However, the global Lipschitz condition, even the local Lipschitz condition, is seemed to be considerably strong when one discusses variable applications in real world. Reference [4] discussed the existence of mild solution to neutral semilinear stochastic functional systems with nonLipschitz coefficients. Reference [5] discussed the existence and uniqueness of solutions to neutral stochastic functional systems with infinite delay under the local Lipschitz conditions in the Euclidean space.

We focus on neutral semilinear stochastic functional dynamic systems for the case where the coefficients do not necessarily satisfy the global Lipschitz condition. Thus we study the existence and uniqueness of mild solutions to neutral semilinear stochastic functional systems with the condition, which was investigated by [6-8] as the Carathéodorytype conditions for strong solutions. Motivated by the above papers, in this paper, we will extend the existence and uniqueness of mild solutions of (2) under the non-Lipschitz conditions to the local non-Lipschitz conditions.
The rest of this paper is organized as follows. In Section 2, we introduce some preliminaries. In Section 3 we prove the existence and uniqueness of the mild solution.

\section{Preliminaries}

Throughout this paper, let $\left(\Omega, \mathscr{F},\left\{\mathscr{F}_{t}\right\}_{t \geq 0}, P\right)$ be a complete probability space with a normal filtration $\left\{\mathscr{F}_{t}\right\}_{t \geq 0}$ satisfying the usual conditions (i.e., it is increasing and right continuous while $\mathscr{F}_{0}$ contains all $P$-null sets). Moreover, let $X, Y$ be two real separable Hilbert spaces, and we denote by $\langle\cdot, \cdot\rangle_{X},\langle\cdot, \cdot\rangle_{Y}$ their inner products and by $\|\cdot\|_{X},\|\cdot\|_{Y}$ their vector norms, respectively. We denote that $L(Y, X)$ denotes the space of all bounded linear operators from $Y$ into $X$, equipped with the usual operator norm $\|\cdot\|$. In this paper, we always use the same symbol $\|\cdot\|$ to denote norms of operators regardless of the spaces potentially involved when no confusion possibly arises. Let $\tau>0$ and $C=C([-\tau, 0] ; X)$ denote the family of all continuous $X$-valued functions $\eta$ from $[-\tau, 0]$ to $X$ with norm $\|\eta\|_{C}=\sup _{t \in[-\tau, 0]}\|\eta(t)\|_{X}$. Here let $C_{\mathscr{F}_{0}}^{b}([-\tau, 0], X)$ be the family of all almost surely bounded, $\mathscr{F}_{0}$-measurable, continuous random variables from $[-\tau, 0]$ to $X$.

Let $\{w(t): t \geq 0\}$ denote a $Y$-valued Wiener process defined on the probability space $\left(\Omega, \mathscr{F},\left\{\mathscr{F}_{t}\right\}_{t \geq 0}, P\right)$ with 
covariance operator Q; that is, $E\langle w(t), x\rangle_{Y} E\langle w(s), y\rangle_{X}=$ $(t \wedge s)\langle Q x, y\rangle_{Y}$, for all $x, y \in Y$, where $Q$ is a positive, selfadjoint, trace class operator on $Y$. In particular, we denote $w(t)$ a $Y$-valued $Q$-Wiener process with respect to $\left\{\mathscr{F}_{t}\right\}_{t \geq 0}$. To define stochastic integrals with respect to the Q-Wiener process $w(t)$, we introduce the subspace $Y_{0}=Q^{1 / 2} Y$ of $Y$ which is endowed with the inner product $\langle u, v\rangle_{Y_{0}}=$ $\left\langle Q^{-1 / 2} u, Q^{-1 / 2} v\right\rangle_{Y}$ and is a Hilbert space. We assume that there exists a complete orthonormal system $\left\{e_{i}\right\}$ in $Y$, a bounded sequence of nonnegative real numbers $\lambda_{i}$ such that $Q e_{i}=\lambda_{i} e_{i}, i=1,2, \ldots$, and a sequence $\left\{\beta_{i}\right\}_{i \geq 1}$ of independent Brownian motions such that

$$
\langle w(t), e\rangle=\sum_{i=1}^{\infty} \sqrt{\lambda_{i}}\left\langle e_{i}, e\right\rangle \beta_{i}(t), \quad e \in Y,
$$

and $\mathscr{F}_{t}=\mathscr{F}_{t}^{w}$, where $\mathscr{F}_{t}^{w}$ is the $\sigma$-algebra generated by $\{w(s): 0 \leq s \leq t\}$. Let $L_{2}^{0}=L_{2}\left(Y_{0}, X\right)$ be the space of all Hilbert-Schmidt operators from $Y_{0}$ to $X$. It turns out to be a separable Hilbert space equipped with the norm $\|u\|_{L_{2}^{0}}^{2}=\operatorname{tr}\left(\left(u Q^{1 / 2}\right)\left(u Q^{1 / 2}\right)^{*}\right)$ for any $u \in L_{2}^{0}$. Obviously, for any bounded operators $u \in L_{2}^{0}$ this norm reduces to $\|u\|_{L_{2}^{0}}^{2}=\operatorname{tr}\left(u Q u^{*}\right)$.

Suppose that $\{S(t), t \geq 0\}$ is an analytic semigroup with its infinitesimal generator $A$; for the literature related to semigroup theory, we suggest Pazy [9]. We suppose $0 \in \varrho(A)$, the resolvent set of $-A$. For any $\alpha \in[0,1]$, it is possible to define the fractional power $(-A)^{\alpha}$ which is a closed linear operator with its domain $\mathscr{D}\left((-A)^{\alpha}\right)$.

Consider the following neutral semilinear stochastic functional systems:

$$
\begin{aligned}
& d\left[x(t)-u\left(t, x_{t}\right)\right]= {\left[A x(t)+f\left(t, x_{t}\right)\right] d t } \\
&+g\left(t, x_{t}\right) d w(t), \quad t \geq 0, \\
& x_{0}(\cdot)=\varphi \in C_{\mathscr{F}_{0}}^{b}([-\tau, 0], X),
\end{aligned}
$$

where $x_{t}=\{x(t+\theta):-\tau \leq \theta \leq 0\}$ can be regarded as a $C([-\tau, 0] ; X))$-valued stochastic process; $u: R_{+} \times$ $C([-\tau, 0], X) \rightarrow X, f: R_{+} \times C([-\tau, 0], X) \rightarrow X, g: R_{+} \times$ $C([-\tau, 0], X) \rightarrow L(Y, X)$ are all Borel measurable; $A$ is the infinitesimal generator of an analytic semigroup of bounded linear operators $S(t), t \geq 0$, in $X$.

Definition 1. A process $\{x(t), t \in[0, T]\}, 0 \leq T<\infty$, is called a mild solution of (2) if

(i) $x(t)$ is adapted to $\mathscr{F}_{t}, t \geq 0$ with $\int_{0}^{T}\|x(t)\|_{X}^{2} d t<$ $\infty$ a.s.;

(ii) $x(t) \in X$ has càdlàg paths on $t \in[0, T]$ a.s.: and, for each $t \in[0, T], x(t)$ satisfies the integral equation,

$$
\begin{aligned}
x(t)= & S(t)[\varphi(0)-u(0, \varphi)]+u\left(t, x_{t}\right) \\
+ & \int_{0}^{t} A S(t-s) u\left(s, x_{s}\right) d s+\int_{0}^{t} S(t-s) f\left(s, x_{s}\right) d s \\
& +\int_{0}^{t} S(t-s) g\left(s, x_{s}\right) d w(s)
\end{aligned}
$$

for any $x_{0}(\cdot)=\varphi \in C_{\mathscr{F}_{0}}^{b}([-\tau, 0], X)$.

To guarantee the existence and uniqueness of a mild solution to (2), the following much weaker conditions, instead of non-Lipschitz condition, are described.

(H1) $A$ is the infinitesimal generator of an analytic semigroup of bounded linear operators $S(t), t \geq 0$, in $X$, and $S(t)$ is uniformly bounded, for some constant $0<a \in R_{+},\|S(t)\| \leq e^{-a t}, t \geq 0$.

(H2) (a) There exists a function $H(t, r): R_{+} \times R_{+} \rightarrow$ $R_{+}$such that $H(t, r)$ is locally integrable in $t \geq 0$ for any fixed $r \geq 0$ and is continuous monotone nondecreasing and concave in $r$ for any fixed $t \in$ $[0, T]$. Moreover, for any fixed $t \in[0, T]$ and $\xi \in X$, the following inequality is satisfied:

$\|f(t, \xi)\|_{X}^{2}+\|g(t, \xi)\|_{L_{2}^{0}}^{2} \leq H\left(t,\|\xi\|_{C}^{2}\right), \quad t \in[0, T]$.

(b) For any constant $K>0$, the differential dynamic system

$$
\frac{d u}{d t}=K H(t, u), \quad t \in[0, T],
$$

has a global solution for any initial value $u_{0}$.

(H3) (a) There exists a function $G(t, r): R_{+} \times R_{+} \rightarrow$ $R_{+}$such that $G(t, r)$ is locally integrable in $t \geq 0$ for any fixed $r \geq 0$ and is continuous monotone nondecreasing and concave in $r$ for any fixed $t \in$ $[0, T] . G(t, 0)=0$ for any fixed $t \in[0, T]$. Moreover, for any fixed $t \in[0, T]$ and $\xi, \eta \in X$, the following inequality is satisfied:

$$
\begin{aligned}
& \|f(t, \xi)-f(t, \eta)\|_{X}^{2}+\|g(t, \xi)-g(t, \eta)\|_{L_{2}^{0}}^{2} \\
& \leq G\left(t,\|\xi-\eta\|_{C}^{2}\right), \quad t \in[0, T] .
\end{aligned}
$$

(b) For any constant $\bar{K}>0$, if a nonnegative function $z(t)$ satisfies that

$$
z(t) \leq \bar{K} \int_{0}^{t} G(s, z(s)) d s, \quad t \in[0, T],
$$

then $z(t)=0$ holds for any $t \in[0, T]$.

(H4) There exist a number $\alpha \in[0,1]$ and a positive $K_{0}$ such that, for any $\xi, \eta \in X$ and $t \geq 0, u(t, x) \in \mathscr{D}\left((-A)^{\alpha}\right)$ and

$\left\|(-A)^{\alpha} u(t, \xi)-(-A)^{\alpha} u(t, \eta)\right\|_{X} \leq K_{0}\|\xi-\eta\|_{C}$.

Moreover, we assume that $u(t, 0)=0$. 
Remark 2. Let $G(t, u)=L(t) \bar{G}(u), t \in[0, T]$, where $L(t) \geq$ 0 is locally integrable and $\bar{G}(u)$ is a concave nondecreasing function from $R_{+}$to $R_{+}$such that $\bar{G}(0)=0, \bar{G}(u)>0$ for $u>$ 0 , and $\int_{0^{+}}(1 / \bar{G}(u)) d u=\infty$. Then by comparison theorem of differential dynamic systems we know that assumption (H3b) holds.

Now let us give some concrete examples of the function $\bar{G}(\cdot)$. Let $\zeta>0$ and let $\delta \in(0,1)$ be sufficiently small. Define

$$
\begin{gathered}
\bar{G}_{1}(u)=\zeta u, \quad u \geq 0, \\
\bar{G}_{2}(u)= \begin{cases}u \log \left(u^{-1}\right), & 0 \leq u \leq \delta, \\
\delta \log \left(\delta^{-1}\right)+\bar{G}_{2}^{\prime}(\delta-)(u-\delta), & u>\delta,\end{cases}
\end{gathered}
$$

where $\bar{G}_{2}^{\prime}$ denotes the derivative of function $\bar{G}_{2}$. They are all concave nondecreasing functions satisfying $\int_{0^{+}}\left(1 / \bar{G}_{i}(u)\right) d u=\infty(i=1,2)$. In particular, we see that Lipschitz condition in [3] and non-Lipschitz conditions in [4] are special cases of our proposed condition.

To show our main results, we need the following lemma.

Lemma 3 (see [9]). If (H1) holds and $0 \in \varrho(A)$, then, for any $\beta \in(0,1]$ :

(i) for each $x \in \mathscr{D}\left((-A)^{\beta}\right)$,

$$
S(t)(-A)^{\beta} x=(-A)^{\beta} S(t) x
$$

(ii) there exist positive constants $M_{\beta}>0$ and $a \in R_{+}$such that

$$
\left\|(-A)^{\beta} S(t)\right\| \leq M_{\beta} t^{-\beta} e^{-a t}, \quad t>0 .
$$

\section{Existence and Uniqueness of the Mild Solution}

In this section, we will establish the existence and uniqueness of the mild solution. When the coefficients $f$ and $g$ satisfy the global Lipschitz condition and the linear growth condition, $[7,8]$ discussed the existence and uniqueness of the mild solution to stochastic differential dynamic systems. In [10], the existence and uniqueness of the mild solution of (2) under the non-Lipschitz condition are given as follows.

Theorem 4. If (H1)-(H4) hold for some $\alpha \in(1 / 2,1]$, then there exists a unique mild solution to (2), provided that

$$
\gamma:=\frac{4 K_{0}^{2} M_{1-\alpha}^{2} a^{-2 \alpha} \Gamma(2 \alpha-1)}{1-K_{0}\left\|(-A)^{-\alpha}\right\|}+K_{0}\left\|(-A)^{-\alpha}\right\|<1,
$$

where $M_{1-\alpha}$ is defined in Lemma 3.

Remark 5. If $G(t, u)=K_{1} u$ for some constant $K_{1}$, then the condition (H3) implies a global Lipschitz condition, which is studied in [3]. In Remark 2, if $L(t)=1$, it is studied in [4]. Therefore, some of the results $[3,4]$ are improved and generalized.
Now, we will replace $(\mathrm{H} 3)$ by the following local nonLipschitz condition.

$\left(\mathrm{H}^{\prime} 3\right)$ (a) For any integer $N>0$, there exists a function $G_{N}(t, r): R_{+} \times R_{+} \rightarrow R_{+}$such that $G_{N}(t, r)$ is locally integrable in $t \geq 0$ for any fixed $r \geq 0$ and is continuous monotone nondecreasing and concave in $r$ with $G_{N}(t, 0)=0$. Moreover, for any fixed $t \in[0, T]$ and $\xi, \eta \in \mathrm{X}$ with $\|\xi\|_{C},\|\eta\|_{C} \leq N$, the following inequality is satisfied:

$$
\begin{gathered}
\|f(t, \xi)-f(t, \eta)\|_{X}^{2}+\|g(t, \xi)-g(t, \eta)\|_{L_{2}^{0}}^{2} \\
\leq G_{N}\left(t,\|\xi-\eta\|_{C}^{2}\right), \quad t \in[0, T] .
\end{gathered}
$$

(b) For any constant $\bar{K}>0$, if a nonnegative function $z(t)$ satisfies that

$$
z(t) \leq \bar{K} \int_{0}^{t} G_{N}(s, z(s)) d s, \quad t \in[0, T],
$$

then $z(t)=0$ holds for any $t \in[0, T]$.

Remark 6. Equation (13) is a generalization of the local Lipschitz condition. $\left(\mathrm{H}^{\prime} 3\right)$ is the local non-Lipschitz condition of this type for the end of wider applications.

Theorem 7. If (H1), (H2), ( $\left.H^{\prime} 3\right)$, and (H4) hold for some $\alpha \in(1 / 2,1]$, then there exists a unique mild solution to (2), provided that

$$
\frac{3 K_{0}^{2} M_{1-\alpha}^{2} a^{-2 \alpha} \Gamma(2 \alpha-1)}{1-K_{0}\left\|(-A)^{-\alpha}\right\|}+K_{0}\left\|(-A)^{-\alpha}\right\|<1,
$$

where $M_{1-\alpha}$ is defined in Lemma 3.

Proof. For any $N \geq 1$, define the truncation functions $f_{N}$ and $g_{N}$ as follows:

$$
\begin{aligned}
& f_{N}\left(t, x_{t}\right)= \begin{cases}f\left(t, x_{t}\right), & \left\|x_{t}\right\|_{C} \leq N, \\
f\left(t, \frac{N x_{t}}{\left\|x_{t}\right\|_{C}}\right), & \left\|x_{t}\right\|_{C}>N,\end{cases} \\
& g_{N}\left(t, x_{t}\right)= \begin{cases}g\left(t, x_{t}\right), & \left\|x_{t}\right\|_{C} \leq N, \\
g\left(t, \frac{N x_{t}}{\left\|x_{t}\right\|_{C}}\right), & \left\|x_{t}\right\|_{C} \leq N,\end{cases}
\end{aligned}
$$

and then the functions $f_{N}$ and $g_{N}$ satisfy (H2) and

$$
\begin{array}{r}
\left\|f_{N}(t, \xi)-f_{N}(t, \eta)\right\|_{X}^{2}+\left\|g_{N}(t, \xi)-g_{N}(t, \eta)\right\|_{L_{2}^{0}}^{2} \\
\leq G_{N}\left(t,\|\xi-\eta\|_{C}^{2}\right), \quad \xi, \eta \in X, \quad t \in[0, T] .
\end{array}
$$


By Theorem 4, there exist the unique mild solutions $x_{N}(t)$ and $x_{N+1}(t)$, respectively, to the following stochastic systems:

$$
\begin{aligned}
x_{N}(t)-u\left(t,\left(x_{N}\right)_{t}\right)= & S(t)[\varphi(0)-u(0, \varphi)] \\
& +\int_{0}^{t} A S(t-s) u\left(s,\left(x_{N}\right)_{s}\right) d s \\
& +\int_{0}^{t} S(t-s) f_{N}\left(s,\left(x_{N}\right)_{s}\right) d s \\
& +\int_{0}^{t} S(t-s) g_{N}\left(s,\left(x_{N}\right)_{s}\right) d w(s), \\
x_{N+1}(t)-u\left(t,\left(x_{N+1}\right)_{t}\right)= & S(t)[\varphi(0)-u(0, \varphi)] \\
& +\int_{0}^{t} A S(t-s) u\left(s,\left(x_{N+1}\right)_{s}\right) d s \\
& +\int_{0}^{t} S(t-s) f_{N+1}\left(s,\left(x_{N+1}\right)_{s}\right) d s \\
& +\int_{0}^{t} S(t-s) \\
& \times g_{N+1}\left(s,\left(x_{N+1}\right)_{s}\right) d w(s) .
\end{aligned}
$$

Define the stopping time $\tau_{N}=T \wedge \inf \left\{t \in[0, T]:\left\|\left(x_{N}\right)_{t}\right\|_{C} \geq\right.$ $N\}$. Recall that, for $a, b \in X, \varepsilon \in(0,1),\|a-b\|_{X}^{2} \leq 1 /(1-$ $\varepsilon)\|a\|_{X}^{2}+1 / \varepsilon\|b\|_{X}^{2}$. Hence, for some $\varepsilon$,

$$
\begin{aligned}
& E \sup _{0 \leq s \leq t \wedge \tau_{N}}\left\|x_{N+1}(s)-x_{N}(s)\right\|_{X}^{2} \\
& \leq \frac{1}{\varepsilon} E \sup _{0 \leq s \leq t \wedge \tau_{N}}\left\|u\left(s,\left(x_{N+1}\right)_{s}\right)-u\left(s,\left(x_{N}\right)_{s}\right)\right\|_{X}^{2} \\
& +\frac{3}{1-\varepsilon} E \sup _{0 \leq s \leq t \wedge \tau_{N}} \| \int_{0}^{s} A S(s-r) \\
& \times\left(u\left(r,\left(x_{N+1}\right)_{r}\right)\right) \\
& \quad-u\left(r,\left(x_{N}\right)_{r}\right) d r \|_{X}^{2} \\
& +\frac{3}{1-\varepsilon} E \sup _{0 \leq s \leq t \wedge \tau_{N}} \| \int_{0}^{s} S(s-r) \\
& \times\left(\left(f_{N+1}\left(r,\left(x_{N+1}\right)_{r}\right)\right.\right. \\
& +\frac{3}{1-\varepsilon} E \sup _{0 \leq s \leq t \wedge \tau_{N}} \| \int_{0}^{s} S(s-r) \\
& \times\left(g_{N+1}\left(r,\left(x_{N+1}\right)_{r}\right)\right. \\
& =: \begin{array}{l}
4 \\
\sum_{i=1} J_{i} .
\end{array}
\end{aligned}
$$

From (H4), we have

$$
\begin{aligned}
J_{1} & \leq \frac{1}{\varepsilon} E \sup _{0 \leq s \leq t \wedge \tau_{N}}\left\|u\left(s,\left(x_{N+1}\right)_{s}\right)-u\left(s,\left(x_{N}\right)_{s}\right)\right\|_{X}^{2} \\
& \leq\left\|(-A)^{-\alpha}\right\|^{2} K_{0}^{2} \frac{1}{\varepsilon} E \sup _{0 \leq s \leq t \wedge \tau_{N}}\left\|\left(x_{N+1}\right)_{s}-\left(x_{N}\right)_{s}\right\|_{C}^{2} \\
& \leq\left\|(-A)^{-\alpha}\right\|^{2} K_{0}^{2} \frac{1}{\varepsilon} E \sup _{0 \leq s \leq t \wedge \tau_{N}}\left\|\left(x_{N+1}\right)(s)-\left(x_{N}\right)(s)\right\|_{X}^{2} .
\end{aligned}
$$

Applying the Hölder inequality, (H4), and Lemma 3, we have

$$
\begin{gathered}
J_{2} \leq \frac{3}{1-\varepsilon} E \sup _{0 \leq s \leq t \wedge \tau_{N}}\left(\int_{0}^{s} \|(-A)^{1-\alpha} S(s-r)(-A)^{\alpha}\right. \\
\times u\left(s,\left(x_{N+1}\right)_{s}\right) \\
\left.-u\left(s,\left(x_{N}\right)_{s}\right) \|_{X} d r\right)^{2} \\
\leq \frac{3}{1-\varepsilon} E \sup _{0 \leq s \leq t \wedge \tau_{N}}\left(\int_{0}^{s} M_{1-\alpha} e^{-a(s-r)}\right. \\
\left.\times \frac{3}{1-\varepsilon} K_{0}^{2} M_{1-\alpha}^{2} a^{-2 \alpha} \Gamma(2 \alpha-r)^{\alpha-1}\left\|(-A)^{\alpha}\right\|_{X} d r\right)^{2} \\
\quad \times E\left(\sup _{0 \leq s \leq t \wedge \tau_{N}}\left\|\left(x_{N+1}\right)(s)-\left(x_{N}\right)(s)\right\|_{X}^{2}\right) .
\end{gathered}
$$

For $0 \leq t \leq \tau_{N}$, we see that $f_{N+1}\left(s,\left(x_{N}\right)_{s}\right)=f_{N}\left(s,\left(x_{N}\right)_{s}\right)=$ $f\left(s,\left(x_{N}\right)_{s}\right)$ and $g_{N+1}\left(s,\left(x_{N}\right)_{s}\right)=g_{N}\left(s,\left(x_{N}\right)_{s}\right)=g\left(s,\left(x_{N}\right)_{s}\right)$. Thus, by $\left(\mathrm{H}^{\prime} 3\right)$ and the Jensen inequality, we obtain

$$
\begin{aligned}
J_{3} \leq \frac{3}{1-\varepsilon} T \int_{0}^{t} G_{N+1}\left(r, E\left(\sup _{0 \leq u \leq r}\right.\right. & \|\left(x_{N+1}\right)(u) \\
& \left.\left.-\left(x_{N}\right)(u) \|_{X}^{2}\right)\right) d r .
\end{aligned}
$$

By $\left(\mathrm{H}^{\prime} 3\right)$, Liu [1, Theorem 1.2.6, page 14] and the Jensen inequality, there exists a positive constant $C_{5}$ such that

$$
\begin{aligned}
J_{4} \leq & \frac{3}{1-\varepsilon} C_{5} \\
& \times \int_{0}^{t} G_{N+1}\left(r, E\left(\sup _{0 \leq u \leq r}\left\|\left(x_{N+1}\right)(u)-\left(x_{N}\right)(u)\right\|^{2}\right)\right) d r .
\end{aligned}
$$


From (19)-(23), choosing $\varepsilon=K_{0}\left\|(-A)^{-\alpha}\right\|$ we have

$$
\begin{aligned}
E \sup _{0 \leq s \leq t \wedge \tau_{N}} & \left\|x_{N+1}(s)-x_{N}(s)\right\|_{X}^{2} \\
\leq & {\left[\left\|(-A)^{-\alpha}\right\| K_{0}\right.} \\
& \left.+\frac{3}{1-K_{0}\left\|(-A)^{-\alpha}\right\|} K_{0}^{2} M_{1-\alpha}^{2} a^{-2 \alpha} \Gamma(2 \alpha-1)\right] \\
& \times E\left(\sup _{0 \leq s \leq t \wedge \tau_{N}}\left\|\left(x_{N+1}\right)(s)-\left(x_{N}\right)(s)\right\|_{X}^{2}\right) \\
& +\left[\frac{3}{1-K_{0}\left\|(-A)^{-\alpha}\right\|} T+\frac{3 C_{5}}{1-K_{0}\left\|(-A)^{-\alpha}\right\|}\right] \\
& \times \int_{0}^{t} G_{N+1}\left(r, E\left(\sup _{0 \leq u \leq r} \|\left(x_{N+1}\right)(u)\right.\right.
\end{aligned}
$$

By (22) and the Gronwall inequality, there exists a constant $\bar{K}$ such that

$$
\begin{aligned}
E \sup _{0 \leq s \leq t} & \left\|x_{N+1}\left(s \wedge \tau_{N}\right)-x_{N}\left(s \wedge \tau_{N}\right)\right\|_{X}^{2} \\
\leq \bar{K} \int_{0}^{t} G_{N+1}\left(r \wedge \tau_{N}, E\left(\sup _{0 \leq u \leq r} \|\right.\right. & \left(x_{N+1}\right)\left(u \wedge \tau_{N}\right) \\
& \left.\left.-\left(x_{N}\right)\left(u \wedge \tau_{N}\right) \|^{2}\right)\right) d r .
\end{aligned}
$$

By $\left(\mathrm{H}^{\prime} 3-\mathrm{b}\right)$, we have

$$
E\left(\sup _{0 \leq s \leq T \wedge \tau_{N}}\left\|x_{N+1}(s)-x_{N}(s)\right\|_{X}^{2}\right)=0, \quad 0 \leq t \leq \tau_{N}
$$

which means thats for $0 \leq t \leq \tau_{N}$, we obtain

$$
x_{N+1}(s)=x_{N}(s) \quad \text { a.e. }
$$

For each $\omega \in \Omega$, there exists an $N_{0}=N_{0}(\omega)>0$ such that $0<T \leq \tau_{N_{0}}$. Define $x(t)$ by $x(t)=x_{N_{0}}(t), t \in[0, T]$. Since $x\left(t \wedge \tau_{N}\right)=x_{N}\left(t \wedge \tau_{N}\right)$, then we have

$$
\begin{aligned}
x\left(t \wedge \tau_{N}\right)-u\left(t, x_{t \wedge \tau_{N}}\right) & \\
= & S(t)[\varphi(0)-u(0, \varphi)] \\
& +\int_{0}^{t \wedge \tau_{N}} A S(t-s) u\left(s, x_{s}\right) d s \\
& +\int_{0}^{t \wedge \tau_{N}} S(t-s) f_{N}\left(s, x_{s}\right) d s
\end{aligned}
$$

$$
\begin{aligned}
& +\int_{0}^{t \wedge \tau_{N}} S(t-s) g_{N}\left(s, x_{s}\right) d w(s) \\
= & S(t)[\varphi(0)-u(0, \varphi)] \\
& +\int_{0}^{t \wedge \tau_{N}} A S(t-s) u\left(s, x_{s}\right) d s \\
& +\int_{0}^{t \wedge \tau_{N}} S(t-s) f\left(s, x_{s}\right) d s \\
& +\int_{0}^{t \wedge \tau_{N}} S(t-s) g\left(s, x_{s}\right) d w(s) .
\end{aligned}
$$

Letting $N \rightarrow \infty$,

$$
\begin{aligned}
x(t)-u\left(t, x_{t}\right)=S( & t)[\varphi(0)-u(0, \varphi)] \\
+ & \int_{0}^{t} A S(t-s) u\left(s, x_{s}\right) d s \\
+ & \int_{0}^{t} S(t-s) f\left(s, x_{s}\right) d s \\
& +\int_{0}^{t} S(t-s) g\left(s, x_{s}\right) d w(s) .
\end{aligned}
$$

Hence we have that $x(t)$ is a mild solution to (2). The proof is complete.

\section{Concluding Remarks}

In the paper, by means of the stopping time technique, the conditions to assure the existence and uniqueness of mild solutions of neutral semilinear stochastic functional dynamic systems are given under the local non-Lipschitz condition, which is a generalization of the local Lipschitz condition. The paper generalizes the results in $[8,10]$.

\section{Acknowledgments}

The work is supported by the Fundamental Research Funds for the Central Universities under Grant 2722013JC080, China Postdoctoral Science Foundation funded project under Grant 2012M511615, the Natural Science Foundation of Hubei Province of China and the National Science Foundation of China.

\section{References}

[1] K. Liu, Stability of Infinite Dimensional Stochastic Differential Equations with Applications, vol. 135, Chapman \& Hall; CRC, London, UK, 2006.

[2] F. Jiang and Y. Shen, "Stability of impulsive stochastic neutral partial differential equations with infinite delays," Asian Journal of Control, vol. 14, no. 6, pp. 1706-1709, 2012.

[3] T. E. Govindan, "Almost sure exponential stability for stochastic neutral partial functional differential equations," Stochastics, vol. 77, no. 2, pp. 139-154, 2005. 
[4] J. Bao and Z. Hou, "Existence of mild solutions to stochastic neutral partial functional differential equations with nonLipschitz coefficients," Computers \& Mathematics with Applications, vol. 59, no. 1, pp. 207-214, 2010.

[5] H. Bao and J. Cao, "Existence and uniqueness of solutions to neutral stochastic functional differential equations with infinite delay," Applied Mathematics and Computation, vol. 215, no. 5, pp. 1732-1743, 2009.

[6] T. Taniguchi, "Successive approximations to solutions of stochastic differential equations," Journal of Differential Equations, vol. 96, no. 1, pp. 152-169, 1992.

[7] J. Turo, "Successive approximations of solutions to stochastic functional-differential equations," Periodica Mathematica Hungarica, vol. 30, no. 1, pp. 87-96, 1995.

[8] B. Xie, "Stochastic differential equations with non-Lipschitz coefficients in Hilbert spaces," Stochastic Analysis and Applications, vol. 26, no. 2, pp. 408-433, 2008.

[9] A. Pazy, Semigroups of Linear Operators and Applications to Partial Differential Equations, Springer, New York, NY, USA, 1992.

[10] F. Jiang and Y. Shen, "A note on the existence and uniqueness of mild solutions to neutral stochastic partial functional differential equations with non-Lipschitz coefficients," Computers \& Mathematics with Applications, vol. 61, no. 6, pp. 1590-1594, 2011. 


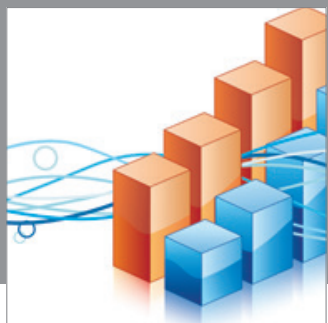

Advances in

Operations Research

mansans

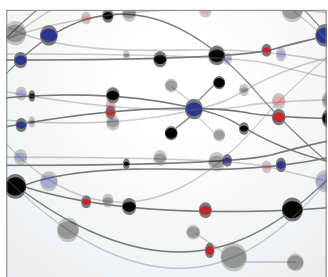

The Scientific World Journal
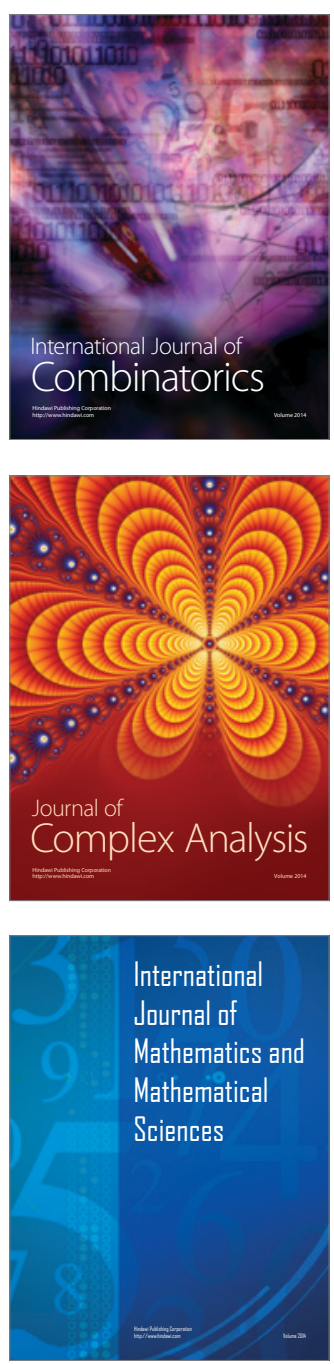
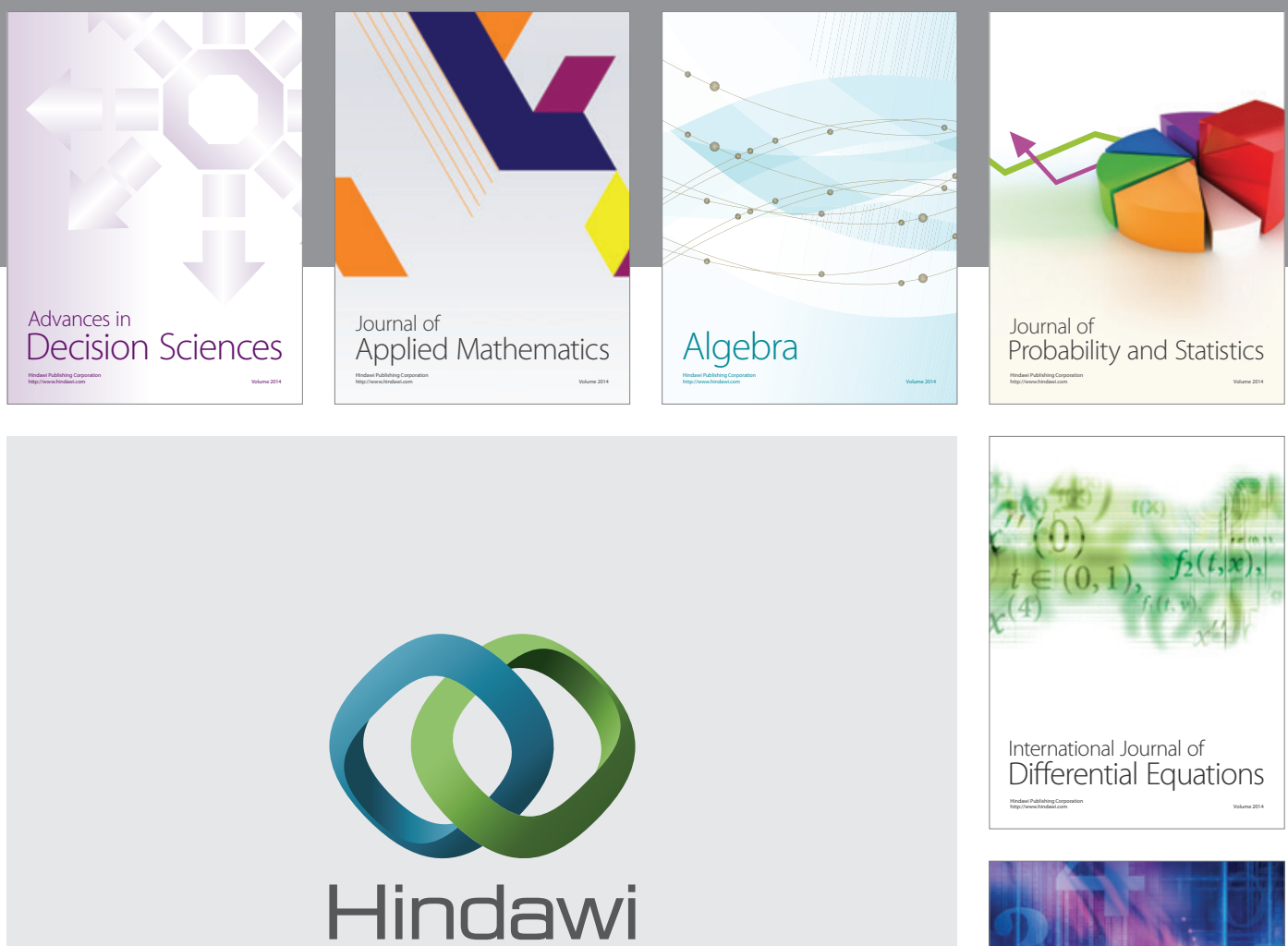

Submit your manuscripts at http://www.hindawi.com
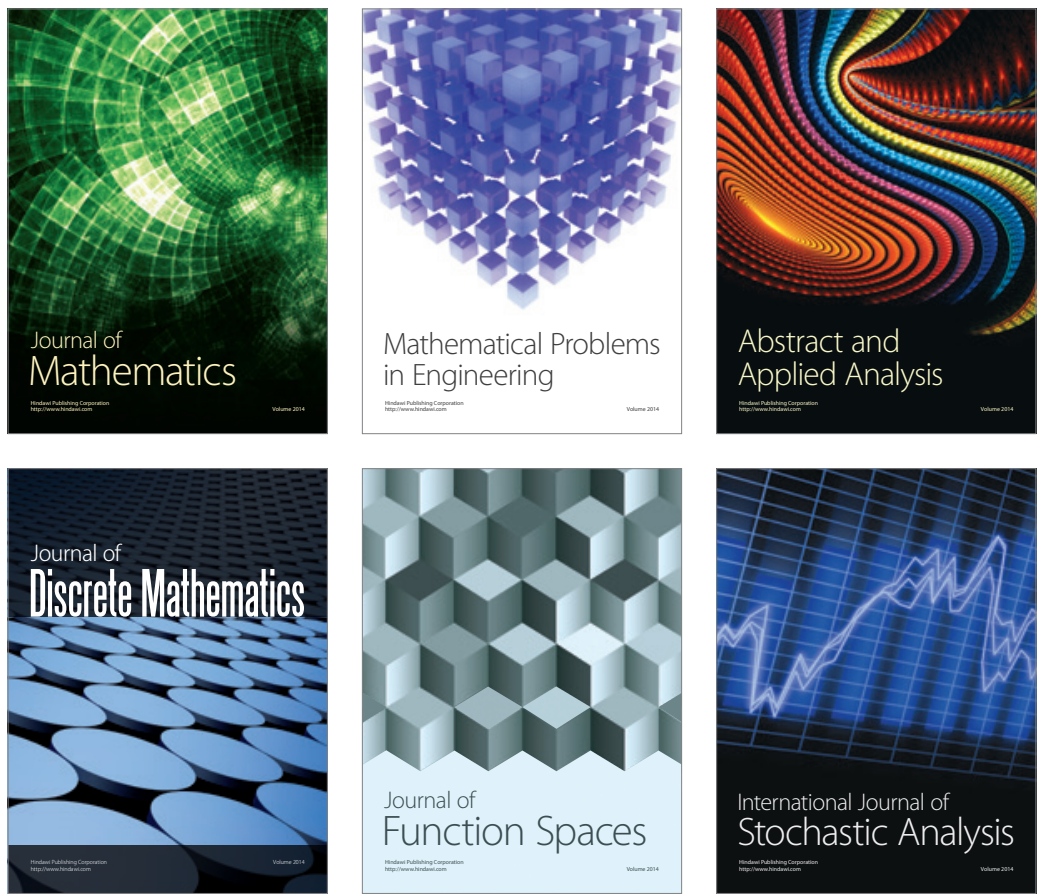

Journal of

Function Spaces

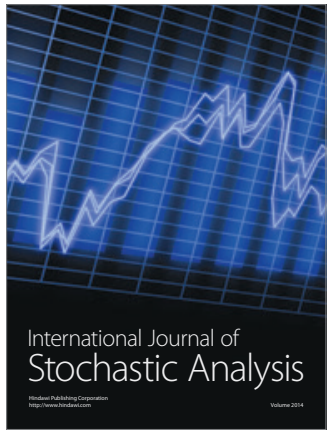

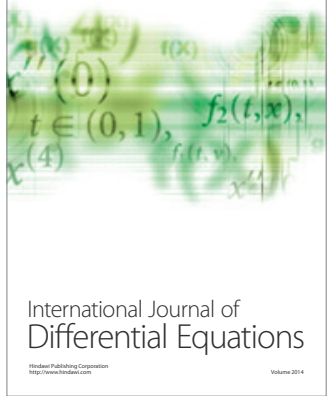
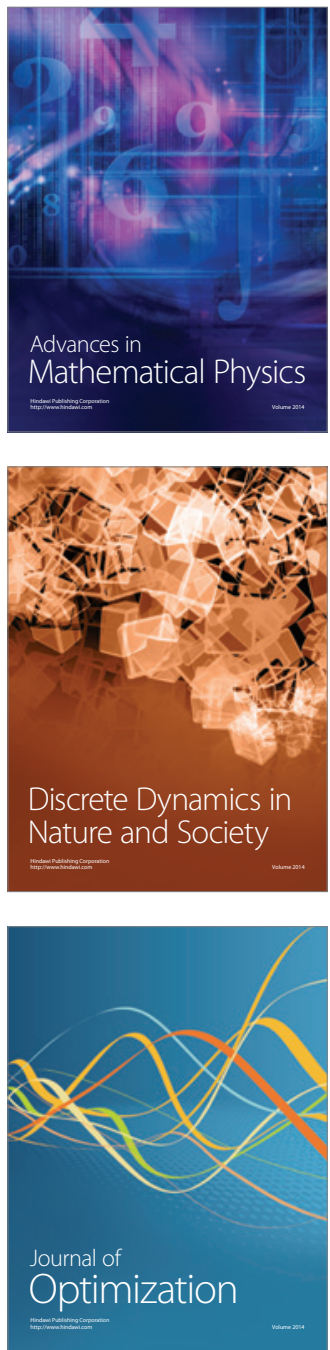